\title{
Message in a Bottle-PEG It Anywhere
}

\author{
Renol Mathew Koshy • Venkata Narasimhan
}

Received: 5 December 2010 /Accepted: 7 December 2010 /Published online: 23 December 2010

(C) Indian Association of Surgical Oncology 2010

Nutrition in head and neck cancer is challenged by swallowing and feeding difficulties due to the disease, poor oral hygiene, treatment side effects and psychosocial factors. The weight loss and treatment induced malnutrition affects nutrition, immunity, quality of life and prognosis. A multi-disciplinary and multi-level involvement is crucial for effective care.

Percutaneous Endoscopic Gastrostomy (PEG) in our country is a grossly misunderstood and underutilized option due to many reasons. A change in attitude towards the PEG can open out an option of enteral feeding which is more physiological, more comfortable, provides a better quality of life with fewer complications.

PEG scores over other tube enterostomies being less invasive compared to the open technique, which is reserved for those who cannot undergo an endoscopy, have an anatomical aberration or as a part of another procedure.

Prophylactic tube enterostomies improve compliance to treatment and hence the outcome.

Predictors of tube enterostomies include disease stage, performance scores and smoking.

\section{The Secondary Hospital Perspective}

Period-Apr 09-Sept 10 (17 months)

Numbers-29 PEGs

Subset of study -15 were for $\mathrm{H} \& \mathrm{~N}$ cancer

Cost-Rs.7000/-(2300-PEG kit + 2700-Procedure + 2000-Hospital stay)

Hospital stay-24hrs

Purpose of stay-To look out for complications and educate about the tube and feeds

Morbidity analysis $(n=13)-1$ major (Necrotizing fasciitis) and 4 minor (peristomal leaks) Duration of PEG-4 months

Follow-up - on OPD basis until removal

Personnel and equipment-Endoscope, a trained General surgeon and an assistant

\section{The Message}

PEG is a very good and cost-effective means of nutritional support in Head and Neck cancer which can safely be done by trained surgeons even in a secondary level hospital. 